\title{
Military Sexual Trauma Survivor Preferences for Provider Gender and Associations With Mental Health Evaluation Attendance
}

\author{
Minden B. Sexton \\ RaeAnn E. Anderson \\ University of North Dakota, raeann.anderson@UND.edu \\ Diana C. Bennett \\ Edward J. Thomas \\ Rachel B. Broman
}

See next page for additional authors

Follow this and additional works at: https://commons.und.edu/psych-fac

Part of the Psychology Commons

\section{Recommended Citation}

Sexton, Minden B.; Anderson, RaeAnn E.; Bennett, Diana C.; Thomas, Edward J.; Broman, Rachel B.; and Richards, Sarah K. H., "Military Sexual Trauma Survivor Preferences for Provider Gender and Associations With Mental Health Evaluation Attendance" (2020). Psychology Faculty Publications. 29. https://commons.und.edu/psych-fac/29

This Article is brought to you for free and open access by the Department of Psychology at UND Scholarly Commons. It has been accepted for inclusion in Psychology Faculty Publications by an authorized administrator of UND Scholarly Commons. For more information, please contact und.commons@library.und.edu. 
Authors

Minden B. Sexton, RaeAnn E. Anderson, Diana C. Bennett, Edward J. Thomas, Rachel B. Broman, and Sarah K. H. Richards 


\title{
Military Sexual Trauma Survivor Preferences for Provider Gender and Associations With Mental Health Evaluation Attendance
}

\author{
Minden B. Sexton, VA Ann Arbor Healthcare System, \\ University of Michigan Medical School \\ RaeAnn E. Anderson, Kent State University, \\ University of North Dakota
}

\author{
Diana C. Bennett, New Mexico Veterans Affairs Healthcare System \\ Edward J. Thomas, Rachel B. Broman, Sarah K. H. Richards, \\ VA Ann Arbor Healthcare System
}

MILITARY SEXUAL TRAUMA (MST) refers to sexual assault or severe sexual harassment experienced during military service. A recent meta-analysis indicated $13.9 \%$ of veterans endorsed MST (38.4\% of women and $3.9 \%$ of men; Wilson, 2018). Although MST is more common in female veterans, given the gender imbalance in military service, men represent a sizable proportion of MST survivors receiving Veterans Health Administration (VHA) care (Kimerling, Street, Gima \& Smith, 2008). Despite nationwide screening efforts and free treatment provided to MST survivors, MST care tends to be underutilized (Kimerling et al., 2015). Approximately half of recent female veterans who are eligible for MSTrelated care access it (Calhoun et al., 2016). Literature among civilian sexual trauma survivors (Kantor, Knefel, Lueger-Schuster, 2017) as well as MST survivors (Turchik, Bucossi, \& Kimerling, 2014; Turchik et al., 2013) suggests that lack of knowledge, stigma, and gender-related issues are large barriers to care. In particular, during the past decade, the U.S. Department of Veterans Affairs has explicitly recommended veterans' preferences for provider gender be assessed and accommodated whenever possible (VHA, 2018). However, there is limited research to date on survivor preferences and whether these are related to treatment-seeking behaviors. For instance, it is possible that articulation of a preference may be associated with more serious consideration of treatment or other factors relevant to engagement. The goal of this study was to investigate the provider gender preferences of male and female U.S. veterans seeking treatment for mental health concerns associated with MST and the association of provider gender preferences with initial visit attendance.

\section{to Treatment Seeking}

Trauma survivors often report intense shame and stigma regarding their experiences (Aakvaag et al., 2016). These emotions are related to increased symptom severity (Aakvaag et al., 2016; Tangney, Wagner, \& Gramzow, 1992) and decreased treatment seeking (Kantor et al., 2017; Sable, Danis, Mauzy, \& Gallagher, 2006). Embarrassment and stigma can be exacerbated for survivors of sexual trauma (Aakvaag et al.), particularly men (Sable et al., 2006). Sexual assault survivors often question their sexuality, masculinity/femininity, self-worth, and relationship functioning after their traumas. For veterans with MST, the unique characteristics of military culture, such as placing high value on masculinity or prioritizing appearances of strength, may further hinder treatment seeking through increased stigma (Bell, Turchik, \& Karpenko, 2014; O’Brien, Keith, \& Shoemaker, 2015). Finally, men often experience sexual violence under different circumstances than women and may experience different clinical outcomes (Anderson, Cahill, \& Delahanty, 2016; Davies, 2002). Trauma-relevant contextual aspects including the increased likelihood of MST perpetration by men, gender-relevant strain, and perception that female clinicians may be less challenging to discuss sensitive issues may relate to careseeking decisions among survivors (e.g., Bell et al., 2014; Elder, Domino, Rentz, \& Mata-Galán, 2017; Monteith, Gerber, Brownstone, Soberay \& Bahraini, 2019). Thus, facilitating care for MST survivors necessitates sensitivity to gender-related issues and potential patient preferences.

The treatment needs and preferences of male sexual trauma survivors are greatly underresearched in comparison to women 
(Donnelly \& Kenyon, 1996; Lowe \& Rogers, 2017; Peterson, Voller, Polusny, \& Murdoch, 2011) and rape myths regarding male survivors continue to abound in spite of legal and policy improvements (Lowe \& Rogers). Of the limited research available on gender and MST treatment, one study of over 4,000 veterans who screened positive for MST found the most powerful factor influencing the receipt of MSTrelated care was gender; male veterans utilized any type of MST-related care less frequently than female veterans in the 1-year period following a positive screen (Turchik, Pavao, Hyun, Mark \& Kimerling, 2012). Thus, understanding the provider gender preferences of both men and women MST survivors may potentially facilitate care, for example, by increasing awareness of VHA system needs for men and women mental MST-skilled health providers.

Complicating this picture, some research suggests PTSD symptoms and psychological distress associated with any diagnosis may increase MST-specific treatment seeking (Calhoun et al., 2016; Kantor et al., 2017), while other research suggests shame and stigma are primary barriers to treatment seeking for people with PTSD (Kantor et al.). Some research suggests female veterans with MST were more likely to endorse PTSD symptoms than their MST-exposed male counterparts (Kimerling et al., 2007), although this finding has less support among veterans seeking mental health treatment for MST-related conditions (Sexton, Raggio, McSweeney, Authier, \& Rauch, 2017). Including PTSD symptom consideration may be helpful for understanding both MST survivors gender preferences for providers and attendance at treatment.

\section{Gender Issues and Treatment Preferences}

Although national VHA policy and clinical tradition recommend accommodating requests for provider gender when possible, there is a mismatch between such recommendations and available data. Research examining therapist-patient gender match tends to show few, if any, significant effects (Lambert, 2016). Research on patient-provider gender match rarely accounts for the gender preferences patients may have. Rather, these studies focus on post-hoc matches or mismatches of gender regardless of patient preferences. Further, this research has rarely included veterans who, due to the unique characteristics of military culture, may have different attitudes and preferences regarding gender issues and treatment (Bell et al., 2014; O'Brien et al., 2015). Further, given the complex relationships between sexual assault trauma, overrepresentation of males among MST perpetrators, and sexual assault and care-seeking stigmas that may influence recovery from trauma among veterans, it may be that therapist gender preference rather than gender matching is critical for engagement in mental health care. Continued research focused on the gender preferences of treatment-seeking veterans endorsing MST appears warranted.

Within veteran populations, research shows facilitating treatment preferences improves treatment satisfaction, completion, and outcomes (Lindheim, Bennett, Trentacosta, \& McLear, 2014) and is consistent with veteran-focused care models. Both quantitative and qualitative research demonstrates female veterans perceive VHA sites that provide gender-sensitive treatment modalities as more accessible and welcoming (Kimerling et al., 2015; Koblinsky, Schroeder, \& Leslie, 2017). For example, female veterans interested in intimate partner violence (IPV)-related care consider the availability of female providers an important facilitator of treatment (Iverson et al., 2016). This data is likely relevant to understanding women seeking MST-specific treatment; female veterans with MST histories often perceive VHA as unwelcoming (Kehle-Forbes et al., 2017). However, much less is known about treatment outcomes and treatment preferences among those seeking care for MSTrelated conditions (Turchik \& Wilson, 2010) compared to patient groups defined by diagnostic status (e.g., veterans with PTSD), female veterans as a whole (Kimerling et al., 2015), or reports related to MST survivors that do not incorporate men.

Despite the prevailing clinical wisdom on gender preferences and VHA policy, only two qualitative, small sample studies researching provider preferences in MST survivors have been published to date. Turchik et al. (2013) interviewed 20 male veterans who had not previously received MST-specific mental health care. Of these, $50 \%$ indicated they would prefer a female clinician, $25 \%$ preferred a male clinician, and $25 \%$ denied a preference. Notably, this study purposefully recruited a sample of male veterans who had screened positive for MST and had utilized some type of VHA care, but had not received any MSTspecific care. In a parallel study with 9 female veterans of whom $55.6 \%$ had attended at least one MST-related visit during the past 2 years, $67 \%$ of women preferred female providers and the remainder had no preference (Turchik, Bucossi, et al., 2014). These preliminary results suggest gender preferences may be quite salient for this population and that preferences may vary by patient gender. However, the small sample size makes it difficult to discern the stability of these rates. Moreover, the use of a non-MST-treatment utilizing male population and women with mixed MST-specific treatment utilization limits our ability to generalize these findings to the needs of those actively considering initiation of MST-specific care. In order to further assess issues related to potential genderspecific needs within liaison to care, Turchik and colleagues expanded on the results of gender-related findings with male veterans (Turchik, Rafie, Rosen, \& Kimerling, 2014). They examined changes in the utilization of mental health care of 153 male veterans following receipt of male-tailored educational materials. Although the materials were reviewed positively, they did not yield an increase in MST-specific mental health utilization.

\section{Current Study}

Prior research with MST treatment seekers shows that, similar to IPV treatment seekers, the gender of the provider is an important concern for patients (Turchik et al., 2013; Turchik, Bucossi, et al., 2014; Turchik, Rafie, et al., 2014). Extant literature suggests male and female MST survivors show a predominant preference for female providers, yet these small sample studies primarily involved qualitative research with veterans not specifically seeking MST-related mental health care. Additionally, none of these studies accounted for potential PTSD symptoms that may prompt treatment seeking or may confound gender-related results. Thus, further research investigating MST survivors' gender preferences using quantitative methods with a larger sample is important for informing VHA policies and practices to optimize MST-related care. The aims of the study were to (a) evaluate MST survivors' gender preferences among a larger sample of veterans accepting referrals for MST-specific care, while accounting for posttraumatic stress symptom screening severity, and (b) to examine relationships between identified preferences, if any, and attendance at consultation appointments with and without controlling for posttraumatic stress disorder screening severity. 


\section{Method}

\section{Participants}

Participants were all 197 veterans endorsing MST who requested referrals for MST-specific mental health care from their VHA clinicians at a Midwestern VHA during an 18-month period between 2015 2016.

\section{Procedures}

As part of VHA standard practices, veterans utilizing health care are screened for MST histories and PTSD symptoms at primary care and other medical visits. For veterans who screened positive, hospital clinicians offered to place consultations to initiate referrals to MST specialists. In addition to MST screening visits, hospital staff were also able to refer to the service based on clinical care needs that may arise after screening or when veterans were more actively interested in pursuing MSTspecific care. The MST consultation service at this VHA was embedded within a PTSD specialty clinic with ability to liaison veterans into outpatient services, such as PTSD interventions, general mental health services, substance use disorder interventions, or behavioral medicine care, following assessment of needs and preferences.

At the time of consultation placement, veterans were asked their preferences (if any) for the gender of their assessing and treating clinician(s) consistent with the local and national policy recommendations. The Military Sexual Trauma Coordinators (MSTC) at the study hospital (one man and one woman) received all consults and facilitated scheduling for a diagnostic evaluation and treatment-planning interviews, hereafter referred to as the MST evaluation, either at the primary outpatient mental health service or through telemental health to the veteran's home or regional community-based outpatient clinics. Veterans seen in Primary Care Mental Health Integration or the Women's Health Clinic had consultation appointments scheduled prior to leaving the screening visit. In cases we were unable to reach a veteran through the above strategies, the MSTC contacted providers associated with upcoming medical visits throughout the hospital to attempt to schedule. Individuals that did not attend initial appointments due to cancellation or no-showing were also contacted, at a minimum, twice by phone and once by mail to attempt to reschedule. Courtesy appointment reminders were sent through the mail or by phone in the week prior to the appointment. Gender preferences were accommodated in all instances and veterans were aware this preference would be honored prior to their appointment. The MSTC monitored whether consultation evaluations were attended and cross-checked this information with the medical record. The hospital's Institutional Review Board approved the processes and procedures involved in this research. As part of a retrospective evaluation of information collected within the purview of standard clinical care at the hospital, the board waived participant consent requirements.

\section{Measures}

Military Sexual Trauma Mental Health Screen and Consultation Instrument. Veterans were screened verbally with the fol- 
lowing items either as part of mandated lifetime screening generally completed in primary care visits, or elsewhere in the hospital when MST was reported: "While you were in the military did you receive uninvited and unwanted sexual attention such as touching, cornering, pressure for sexual favors, or verbal remarks?" and "While you were in the military did someone ever use force or the threat of force to have sexual contact with you against your will?" Veterans who indicated "yes" to either of these items were then asked whether they were interested in speaking to a provider for MST-specific care, and affirmative answers triggered a referral to an MST specialist. Providers generating consultation requests for the MST team were required to select whether the veteran preferred a male clinician, female clinician, or had no preference in order to submit the consult to the service.

Primary Care-PTSD Screen (PC-PTSD). The PC-PTSD (Prins, Ouimette, \& Kimerling, 2003) is a four-item screen developed based on Diagnostic and Statistical Manual of Mental Disorders IV-TR (American Psychiatric Association, 2000) PTSD symptoms for rapid use in medical settings. The assessment cues for potentially traumatic

Table 1. Veteran Demographic, Service, and PTSD Screening Characteristics $(N=195)$

\begin{tabular}{lcc}
\hline Characteristic & \% or Mean & SD \\
\hline Age & 44.92 & 13.37 \\
Women & $73.6 \%$ & \\
Caucasian & $69.2 \%$ & \\
African American & $24.4 \%$ & \\
American Indian & $2.3 \%$ & \\
Latina/Latino & $1.7 \%$ & \\
Married & $26.6 \%$ & \\
Service Branch & & \\
$\quad$ Air Force & $15.2 \%$ & \\
$\quad$ Army & $49.3 \%$ & \\
Marines & $5.1 \%$ & \\
$\quad$ Navy & $30.4 \%$ & \\
Service Era & & \\
Vietnam & $14.7 \%$ & \\
Post-Vietnam & $20.6 \%$ & \\
Persian Gulf & $24.1 \%$ & \\
$\quad$ OIF/OEF/OND & $40.6 \%$ \\
PC-PTSD & 2.01 & 1.75 \\
\hline
\end{tabular}

Notes. OIF/OEF/OND = Operation Iraqi Freedom/Operation Enduring Freedom/Operation New Dawn; PCPTSD = Primary Care PTSD Screen. events, and past-month endorsement of three or more symptoms (e.g., having nightmares, trying hard to avoid thoughts and situations related to the event) is considered a positive screen indicating further attention to the assessment and management of PTSD may be warranted. The PCPTSD has been psychometrically supported for use with active-duty service members and veterans (Bliese et al., 2008; Calhoun et al., 2010; Ouimette, Wade, Prins, \& Schohn, 2008).

\section{Data Analysis}

Demographic characteristics were assessed with descriptive statistics as appropriate. Associations between patient gender, provider gender preference, and evaluation attendance were examined using chi-square analyses. Logistic regression was used to evaluate the potential main effects of patient gender, gender preference, and PC-PTSD and the interaction of patient gender and gender preference with evaluation attendance. Phi coefficients were used to characterize the magnitude of significant effects. All analyses were performed with SPSS 24.0.

\section{Results}

\section{Participant Characteristics}

One hundred ninety-seven veterans had consultations placed for care during the study. Only $1 \%$ ( $n=2$; one man and one woman) of veterans expressed a preference for a male provider and, thus, these two cases were removed from further analyses due to the small sample size of this group. Regarding the remaining 195 participants, they were primarily women $(73.3 \%)$. Veterans identified as Caucasian non-Hispanic (68.7\%), African American (23.1\%), American Indian (2.1\%), Latino or Latina (1.5\%), or another ethnoracial group/biracial/did not identify (4.6\%). The average age was 45.2 years. Men comprised approximately a quarter of those seeking MSTspecific treatment (26.4\%), notably lower than the representation of males within the MST-positive population receiving any services at this VHA (44.5\%). Most were not married, and a variety of service eras and branches were represented. See Table 1 .

\section{Gender Preferences}

Overall, $47.2 \%$ of participants requested a female clinician, with the remaining having no gender preference. Among female veterans, $53.5 \%$ requested a woman in contrast with $29.4 \%$ of men. The patient gender difference in provider gender preference was significant $\left(\chi^{2}=8.75, d f=1, p=\right.$ $.003)$. The magnitude of the effect was small-to-medium $(\Phi=.213)$. The PCPTSD was not associated with gender preference $(t=0.129, d f=158, p=.890)$.

\section{Gender and Gender Preference Associa- tions With Evaluation Attendance}

The rate of attendance at MST-specific evaluation appointments was $73.6 \%$. Attendance rates were $74.6 \%$ and $70.6 \%$ for women and men, respectively. This difference was not statistically significant $\left(\chi^{2}=\right.$ $0.32, d f=1, p=.573)$. Specific to gender preferences, requesting a female provider was associated with an $80.2 \%$ attendance rate while those indicating no gender preference demonstrated a $67.6 \%$ attendance rate. This comparison was statistically significant $\left(\chi^{2}=3.91, d f=1, p=.048\right)$ though the magnitude of the effect was small $(\Phi=$ .142). See Figure 1.

Given the significant relationship between patient gender and provider gender preference observed, we evaluated whether the predictive utility of provider gender preference on attendance could be improved with incorporating patient gender, the interaction of patient gender and gender preference, and PC-PTSD scores into the model. Logistic regression found patient gender $(p=.549)$, the patient gender and preference interaction $(p=$ $.378)$, and PC-PTSD scores (.962) did not improve the model $\left(\chi^{2}=4.83, d f=3, p=\right.$ $.185)$.

\section{Discussion}

To our knowledge, the current study was the first to examine how veterans' preference for provider gender is related to attendance at MST-specific mental health evaluation visits. Further, whereas women were more likely to articulate a preference for female providers, having a preference for a female provider (versus no gender preference) was associated with greater likelihood to attend visits, while female gender of the veteran was not.

Approximately half of women and one third of men articulated a preference for female providers. However, our results were only partially consistent with those reported in previous research with smaller samples of veterans who were not seeking MST-specific mental health care (Turchik et al., 2013; Turchik, Bucossi, et al., 2014). Regarding female veterans' preferences, our results roughly parallel Turchik's interviews with women; $50 \%$ of veterans in this 
study requesting female providers in contrast with $67 \%$ in Turchick, Bucossi, et al. (2014). Our results differ more substantially from Turchik et al.'s (2013) study of male veterans. Our study identified a notable majority of male veterans $(69.6 \%)$ did not have a preference for provider gender in contrast with $25 \%$ in the qualitative study; the qualitative report indicated $50 \%$ of male respondents preferred a female and $25 \%$ a male, and we observed $29.4 \%$ of men requesting a female provider and only a single male veteran requesting a male clinician. There may be several reasons for these discrepancies. First, the Turchik studies were based on a very small number of participants, a history of MSTrelated mental health care was an exclusion criterion for men, and a sizeable minority of female veterans were not treatment seeking. In contrast, our study focused exclusively on those who requested or accepted referrals for MST-specific mental health care. It may be that the observed variability reflects differences between active MST treatment seekers and non-treatmentseekers or greater consideration of preferences when accepting an evaluation oppor- tunity. In addition, it may be that male veterans who prefer male clinicians may have additional, unresearched factors that reduce willingness to even accept an MSTrelated consultation. Further research evaluating intersections between veteran gender and military culture with other aspects of diversity would be illustrative.

The overall attendance rate for the MST evaluation was encouraging. However, nearly a quarter of veterans were lost to care between the placement of the consultation and the evaluation, despite the frequent outreach contact provided by the hospital to establish first visits and reschedule missed appointments. Our study found veteran gender was not associated with completion of evaluations, though those who requested a female provider were more likely to attend than those who indicated they did not have a provider preference. It is possible that those who select a provider gender preference may be less likely to be in precontemplative or contemplative stages regarding engaging in treatment and that active selection of a provider characteristic signals greater consideration 
of readiness for care entry (Norcross, Krebs \& Prochaska, 2011).

The current study has a number of strengths. Our sample included all veterans for whom an MST-related mental health consult was placed within the studied time frame, with sufficient numbers of both male and female veterans, which may improve the generalizability of our findings. Further, the utilization of participants actively seeking care clarifies potential preference differences in those seeking treatment in contrast to samples included in the limited previous research.

Although we used a sample of treatment-seeking veterans that should be highly generalizable, we were limited in the number requesting a male provider. We were also unable to follow up with those who did not attend to determine what factors may have contributed. We were limited in our ability to collect other variables that may have influenced attendance, such as readiness for treatment, psychosocial stressors, or history of treatment seeking outside of our VHA clinic. Although we included PC-PTSD score, this is only a screening measure, and full indices of symptom severity were unavailable for those who did not attend the appointment. Further, our participants were limited to veterans who disclosed MST to their provider and accepted a mental health referral for care. Of particular importance to our study, it should be noted that continuing research identifies that male veterans, in particular, may be less apt to disclose MST through national VHA screening procedures (Bovin et al., 2019)

There are likely a variety of factors influencing gender preference and these underlying characteristics remain poorly understood. For instance, an individual's history of treatment experiences or contextual aspects of the assault, such as the gender of perpetrators, may be important factors in determining when an individual would request a provider of a certain gender (Turchik et al., 2013). Empirical examination of these and other factors are clear next steps in furthering our understanding of role of gender preference in treatment outcomes for MST survivors. A greater understanding of these factors will allow providers to better facilitate treatment engagement. It was further surprising that scores on the PC-PTSD screen did not improve the fit of our models. Although PTSD is only one of several common outcomes following trauma, one might anticipate that those reporting greater pastmonth stress would be more likely to present for care. Further attention to aspects of clinical care management and engagement in the context of MST with elevated PTSD symptoms would be illustrative and may identify additional pathways to assist survivors who are screening positive for current stress. Additionally, future work should examine how gender preference may relate to use and completion of evidence-based psychophamacology and psychotherapy resources for MSTrelated conditions as well as potential relationships with clinical outcomes. Previous work has identified that a gender match between patient and clinician is not, itself, related to psychotherapy retention for PTSD treatment, and in fact a gender match for male patients may be a negative predictor of treatment retention (Shiner, Westgate, Harik, Watts, \& Schnurr, 2017). This suggests the relationship between patient and clinician gender is complex and gender preference may be more important than gender match. However, the relationship between patient-provider gender preference match and clinical outcome has not been previously evaluated with MST survivors. Although certainly not all MST survivors have mental health symptoms significant enough to warrant treatment, future studies are also needed to determine ways in which more veterans with active MST-related symptoms can be engaged in care and to identify barriers to accepting a referral for treatment.

\section{Implications for Policy or Practice}

It is recommended that VHA mental health service teams ensure composition of sufficiently trained staff in MST and trauma-focused care such that preferences can be honored whenever possible. There may be a subpopulation of male veterans who would prefer a male clinician (as evidenced in Turchik's study of non-treatment-seekers) who are, as yet, declining mental health referrals for MST. Further, the percentage of veterans in this study expressing no preference for provider gender may serve to allay misconceptions that veterans will reject male clinicians for MST-focused interventions.

The observation of more limited use of care by male veterans, even after controlling for PTSD symptoms reported at screening, is concerning as significant efforts have been made during recent years to raise awareness of MST services and address barriers for male veterans at a national and local level. At the local level, male-specific brochures about MST and associated care were disseminated throughout the hospital and a male MST Coordinator was available during this time. Nationally, the VHA requires service providers to receive MST training to augment skills when identifying and treating survivors that incorporates awareness of MST and its relationships to physical and mental health and facilitated sensitive screening, referral, and appropriate care related to their service role (VHA, 2018).

Given that a positive MST history is associated with increased hospital service utilization (e.g., Mercado, Wiltsey-Sterman, \& Iverson, 2015), our results support the notion that additional research on barriers to treatment, particularly for male survivors, is needed. A qualitative study by Turchik et al. (2013) identified common barriers to treatment seeking for male survivors of MST include unhelpful beliefs about masculinity, sexuality, and gender roles that may be specific to men; these challenges are often more pronounced in military populations (Bell et al., 2014; O'Brien et al., 2015). It is possible that some of these impediments may be addressed through conversations with veterans at the time of MST screening and referral for treatment. However, as non-mental-health providers commonly conduct these screenings, additional training may be for such clinicians to reduce potential barriers to disclosing MST (e.g., Andersen \& Blais, 2018) and accepting referrals for treatment.

In addition, further hospital utilization mapping of the care trajectories of mental and non-mental-health VHA use for male veterans with MST is advised to identify the degree to which MST-related care may be indicated and augment policies to better target issues associated with nonengagement in care. For instance, MST screening is generally conducted during first visits with primary care providers. It may be the case that, as veterans increase familiarity with care providers and establish trusting relationships over time, they may be more open to consideration of MST-specific care through VHA. Currently, MST screenings and associated consultation questions are designated as a single lifetime clinical reminder. It may be helpful to consider, for those with lifetime positive screens, to have reminder mechanisms in place to inquire about interest in care liaison on a recurrent basis or to consider repeat screenings to better capture those that may be more likely to have false negatives on MST screens when newly entering the VHA system. 


\section{References}

Aakvaag, H. F., Thoresen, S., WentzelLarsen, T., Dyb, G., Røysamb, E., \& Olff, M. (2016). Broken and guilty since it happened: A population study of trauma-related shame and guilt after violence and sexual abuse. Journal of Affective Disorders, 204, 16-23.

American Psychiatric Association. (2000). Diagnostic and statistical manual of mental disorders (4th ed.). Washington, DC: Author.

Anderson, R. E., Cahill, S. P., \& Delahanty, D. L. (2016). The psychometric properties of the Sexual Experiences SurveyShort Form Victimization (SES-SFV) and characteristics of sexual victimization experiences in college men. Psychology of Men \& Masculinity, 19, 25-34.

Andresen, F. J., \& Blais, R. K. (2018). Higher self-stigma is related to lower likelihood of disclosing military sexual trauma during screening in female veterans. Psychological Trauma: Theory, Research Practice, \& Policy, 22, 372-378.

Bell, M. E., Turchik, J. A., \& Karpenko, J. A. (2014). Impact of gender on reactions to military sexual assault and harassment. Health \& Social Work, 39, 25-33.

Bliese, P. D., Wright, K. M., Adler, A. B., Cabrera, O., Castro, C. A., \& Hoge, C. W. (2008). Validating the Primary Care Posttraumatic Stress Disorder screen and the Posttraumatic Stress Disorder Checklist with soldiers returning from combat. Journal of Consulting and Clinical Psychology, 76, 272.

Bovin, M. J., Black, S. K., Kleiman, S. E., Brown, M. E., Brown, L. G., Street, A. E., ... Marx, B. P. (2019). The impact of assessment modality and demographic characteristics on endorsement of military sexual trauma. Women's Health Issues, 29, S67-S73.

Calhoun, P. S., McDonald, S. D., Guerra, V. S., Eggleston, A. M., Beckham, J. C., Straits-Troster, K., \& VA Mid-Atlantic MIRECC OEF/OIF Registry Workgroup. (2010). Clinical utility of the Primary Care-PTSD Screen among US veterans who served since September 11, 2001. Psychiatry Research, 178, 330-335.

Calhoun, P. S., Schry, A. R., Dennis, P. A., Wagner, H. R., Kimbrel, N. A., Bastian, L. A., ... Straits-Tröster, K. (2016). The association between military sexual trauma and use of VA and non-VA health care services among female veterans with military service in Iraq or Afghanistan. Journal of Interpersonal Violence, 42, 269-287.

Davies, M. (2002). Male sexual assault victims: A selective review of the literature and implications for support services.
Aggression and Violent Behavior, 7, 203214.

Donnelly, D. A., \& Kenyon, S. (1996).

"Honey, we don't do men" gender stereotypes and the provision of services to sexually assaulted males. Journal of Interpersonal Violence, 11, 441-448.

Elder, W. B., Domino, J. L., Rentz, T. O., \& Mata-Galán, E. L. (2017). Conceptual model of male military sexual trauma. Psychological Trauma: Theory, Research, Practice, and Policy, 9(S1), 59.

Iverson, K. M., Stirman, S. W., Street, A. E., Gerber, M. R., Carpenter, S. L., Dichter, M. E., ... Vogt, D. (2016). Female veterans' preferences for counseling related to intimate partner violence: Informing patient-centered interventions. General Hospital Psychiatry, 40, 33-38.

Kantor, V., Knefel, M., \& Lueger-Schuster, B. (2017). Perceived barriers and facilitators of mental health service utilization in adult trauma survivors: A systematic review. Clinical Psychology Review, 52, 52-68.

Kehle-Forbes, S. M., Harwood, E. M., Spoont, M. R., Sayer, N. A., Gerould, H., \& Murdoch, M. (2017). Experiences with VHA care: A qualitative study of U.S. women veterans with self-reported trauma histories. BMC Women's Health, $17,38$.

Kimerling, R., Gima, K., Smith, M. W., Street, A., \& Frayne, S. (2007). The Veterans Health Administration and military sexual trauma. American Journal of Public Health, 97, 2160-2166.

Kimerling, R., Pavao, J., Greene, L., Karpenko, J., Rodriguez, A., Saweikis, M., \& Washington, D. L. (2015). Access to mental health care among women veterans: Is VA meeting women's needs? Medical Care, 53, S97-S104.

Kimerling, R., Street, A. E., Gima, K., \& Smith, M. W. (2008). Evaluation of universal screening for military-related sexual trauma. Psychiatric Services, 59, 635-640.

Koblinsky, S. A., Schroeder, A. L., \& Leslie, L. A. (2017). "Give us respect, support and understanding": Women veterans of Iraq and Afghanistan recommend strategies for improving their mental health care. Social Work in Mental Health, 15, 121-142.

Lambert, M. J. (2016). Does client-therapist gender matching influence therapy course or outcome in psychotherapy. Evidence Based Medicine and Practice, 2, 2.

Lindheim, O., Bennett, C. B., Trentacosta, C. J., \& McLear, C. (2014). Client preferences affect treatment satisfaction, completion, and clinical outcome: A meta- analysis. Clinical Psychology Review, 34, 506-517.

Lowe, M., \& Rogers, P. (2017). The scope of male rape: a selective review of research, policy and practice. Aggression and Violent Behavior, 35, 38-43.

Mercado, R. C., Wiltsey-Stirman, S. Iverson, K. M. (2015). Impact of childhood abuse on physical and mental health status and health care utilization among female veterans. Military Medicine, 180, 1065-1074.

Monteith, L. L., Gerber, H. R., Brownstone, L. M., Soberay, K. A., \& Bahraini, N. H. (2019). The phenomenology of military sexual trauma among male veterans. Psychology of Men \& Masculinities, 20(1), 115.

Norcross, J. C., Krebs, P. M., \& Prochaska, J. O. (2011). Stages of change. Journal of Clinical Psychology, 67, 143-154.

O'Brien, C., Keith, J., \& Shoemaker, L. (2015). Don't tell: Military culture and male rape. Psychological Services, 12, 357-365. http://doi.org/10.1037/ ser0000049

Ouimette, P., Wade, M., Prins, A., \& Schohn, M. (2008). Identifying PTSD in primary care: Comparison of the Primary Care-PTSD screen (PC-PTSD) and the General Health Questionnaire-12 (GHQ). Journal of Anxiety Disorders, 22, 337-343.

Peterson, Z. D., Voller, E. K., Polusny, M. A., \& Murdoch, M. (2011). Prevalence and consequences of adult sexual assault of men: review of empirical findings and state of the literature. Clinical Psychology Review, 31, 1-24.

Prins, A., \& Ouimette, P., \& Kimerling, R. (2003). The primary care PTSD screen (PC-PTSD): Development and operating characteristics. Primary Care Psychiatry, 9, 151

Sable, M. R., Danis, F., Mauzy, D. L., \& Gallagher, S. K. (2006). Barriers to reporting sexual assault for women and men: Perspectives of college students. Journal of American College Health, 55, 157-162.

Sexton, M. B., Raggio, G. A., McSweeney, L. B., Authier, C. C., \& Rauch, S. A. M. (2017). Contrasting gender and combat versus military sexual traumas: Psychiatric symptom severity and morbidities in treatment-seeking veterans. Journal of Women's Health, 26, 933-940.

Shiner, B., Westgate, C. L., Harik, J. M., Watts, B. V., \& Schnurr, P. P. (2017). Effect of patient-therapist gender match on psychotherapy retention among United States veterans with posttraumatic stress disorder. Administration and Policy in Mental Health and Mental Health Services Research, 44, 642-650. 
Tangney, J. P., Wagner, P., \& Gramzow, R. (1992). Proneness to shame, proneness to guilt, and psychopathology. Journal of Abnormal Psychology, 101, 469478.

Turchik, J. A., Bucossi, M. M., \& Kimerling, R. (2014). Perceived barriers to care and gender preferences among veteran women who experienced military sexual trauma: A qualitative analysis. Military Behavioral Health, 2, 180-188.

Turchik, J. A., McLean, C., Rafie, S., Hoyt, T., Rosen, C. S., \& Kimerling, R. (2013). Perceived barriers to care and provider gender preferences among veteran men who have experienced military sexual trauma: A qualitative analysis. Psychological Services, 10, 213-222.

Turchik, J. A., Pavao, J., Hyun, J., Mark, H., \& Kimerling, R. (2012). Utilization and intensity of outpatient care related to military sexual trauma for veterans from Afghanistan and Iraq. Journal of Behavioral Health Services and Research, 39, 220-233.

Turchik, J. A., Rafie, S., Rosen, C. S., \& Kimerling, R. (2014). Preferences for gender-targeted health information: a study of male veterans who have experienced military sexual trauma. American Journal of Men's Health, 8, 240-248.

Turchik, J. A., \& Wilson, S. M. (2010, July 1). Sexual assault in the U.S. military: A review of the literature and recommendations for the future. Aggression and Violent Behavior, 15, 267-277.

Veterans Health Administration. (2018). VHA Directive 115: Military sexual trauma (MST) program. Department of Veterans Affairs, Washington, DC.

Wilson, L. C. (2018). The prevalence of military sexual trauma: a meta-analysis. Trauma, Violence, \& Abuse, 19(5), 584597.

This study was supported by the Ann Arbor Veterans Healthcare System Mental Health Service and the University of Michigan Department of Psychiatry. The contents do not necessarily represent the views of the U.S. Department of Veterans Affairs or the United States Government.

The authors have no conflicts of interest to report.

Correspondence to Minden B. Sexton, Ph.D., Ann Arbor Veterans Healthcare Administration, Mental Health Service (116C), 2215 Fuller Rd., Ann Arbor, 48105; 